\title{
Simplification Method and Application of 3D Laser Scan Point Cloud Data
}

\author{
GeFang Wang \\ Mechanical Technology Research Institute \\ Mechanical Engineering College \\ Shi Jiazhuang, China \\ haning1103@163.com \\ Ning Han \\ Mechanical Technology Research Institute \\ Mechanical Engineering College \\ Shi Jiazhuang, China
}

\author{
YanMei Lv \\ Mechanical Technology Research Institute \\ Mechanical Engineering College \\ Shi Jiazhuang, China \\ Dong Zhang \\ Mechanical Technology Research Institute \\ Mechanical Engineering College \\ Shi Jiazhuang, China
}

\begin{abstract}
The density of acquired data by 3D laser scanner is huge and is not convenient for curved face reconstruction, model reconstruction, data storage, data display and transmission. Firstly, the conventional point cloud data classification and simplification method is introduced. Secondly, the angle deviation error and chord deviation error simplification method is researched based on the analysis of characteristic of 3D laser scan point cloud data. Finally, the angle--chord comprehensive simplification method is prompted. The example shows that this method could simplify the scan line point cloud data got by $3 D$ laser scanner directly and effectively. This method could retain the point cloud data which reflects the surface character of object with higher simplification efficiency.
\end{abstract}

Keywords-laser scan; point cloud data; data simplication; angle deviation error; chord deviation data;

\section{INTRODUCTION}

With the rapid development of the three laser scanning technology, people can quickly obtain a large number of product surface data. Measurement using a 3D laser scanner, each scanned point cloud data based on tens of thousands of massive data, contains more information, but the point cloud density. The huge cloud of data points to Surface Reconstruction model reconstruction and subsequent processing and storage, display and transmission inconvenience. Instead the one hand, not all of the data points in the follow-up process can be used each year in the area of curvature smaller at too dense cloud of data points that may affect the surfaces of the Guangshun sexual and reconstruction accuracy; another party sensitive, too the dense point cloud will not only increase the amount of computation, processing speed slows down, spend a lot of time, at the same time as data storage, display and transmission will consume a large amount of computer resources. Therefore, there is need to ensure that streamline the handling of mass data point clouds under the premise of a certain accuracy, extract the data point clouds reflected in the surface shape of the data points, removing a lot of redundant data points.

\section{LASER SCANNING DATA POINT PlOUd TO STREAMLine METHODS}

\section{A. Point cloud to streamline methods for commonly used data}

Usually referred to as the set of data points obtained in three-dimensional space of the three-dimensional coordinate measuring point cloud, the smallest point cloud contains only one point the point cloud of the high density of up to several million data points. According to the principle of data reduction and the effect of different commonly used data point cloud streamline method can be divided into the following three categories:

(1) Simplification method based on distance. Such methods are actually the size of the distance between adjacent data points with a little rain as a basis to determine whether to delete the store. The characteristics of this method is relatively simple algorithms and mathematical expression, little change in curvature of the data point cloud streamlining better, but the change in curvature, a mutation in the data point cloud streamline effect is poor.

(2) Curvature class streamline method. Class method is based on the removal of a point to change the size of the surface curvature as the basis to determine whether or not to remove the. Class delete the point at the change of curvature large than small curvature change less, because the change in curvature large to delete little after causing curvature change is larger than the curvature of the small, so the law can change in curvature large remained relatively better results.

(3) Uniform classes streamlining method. The type of method is to remove the excess point within a predetermined space (quadrilateral, square or spherical) to retain only one point, like the principle of "median filtering". Class method streamlining speed fast, simple algorithm, streamline in gentle curvature change Annex characteristics cases better, faster change in curvature when the error is larger. 
Different types of data in order to effectively streamline the processing of various types of data point cloud, point cloud usually need to streamline the method using different algorithms. The: scattered cloud points generally distance classes to streamline the handling of scan line data point cloud can be tired by curvature streamline gridded data point cloud should adopt a uniform class streamline method.

\section{B. 3D laser scanning data point cloud characteristics}

The basic principle is that of the non-contact laser triangulation method (three-dimensional laser scanning method) having a regular geometric shape of the laser light source (such as: a point source, line source) projected onto the surface of the measured object, the three-dimensional object plane type spatial modulation of the beam production, changing the angle of the imaging beam, and forming the diffuse reflection points of light (or light band) is placed in a position in space of the image sensor (CCD) imaging, the imaging point position is immediately changed, in accordance with the triangular principle to calculate the measured point space coordinates. This method of measurement has a measurement speed, high efficiency, a method is currently the most widely used, the most mature technology.

The actual three-dimensional data measurement process, the gridded data point cloud is generally difficult or too few to several non-contact laser scanner obtained data from gridded data point cloud, most of the laser scanner measurements the data obtained are often scan line data point cloud. Scan-line data on the appearance of the point cloud is rendered as one of the scan lines, a sweep surface lines by the laser probe head to move in a straight line on the prime minister, because each point in the process of moving are sequentially generated, therefore, each of the scanning line point in accordance with its spatial position are arranged in a linked list of stored data, in addition, the movement characteristics of the laser probe head along a linear direction so that these points are located within the same plane, make full use of the above two important characteristics of the data point cloud of laser scanning can be effectively achieved streamlining processing.

\section{Angle - chord comprehensive simplification method}

Scan line data point cloud to streamline common use of angle deviation method, chord deviation bounding box method, the distance law streamline paper mainly discusses curvature changes to streamline point cloud angle deviation method chords deviation method (shown in Figure 1).



Figure1. Basic principle of angle deviation error method and chord deviation error method.

Angle deviation method and works as follows: For a certain point of the scanning line (both ends except) $\mathrm{Pi}$ between, store adjacent thereto highlights constitute a reflecting surface to the angle deviation of a vector, between the two vectors the point of the curvature change, which can decide whether to remove the point according to the size of the chord height deviation.

However, if used alone angle deviation method for data reduction, timely angle between adjacent bright spot together far bow may be significant, then can not simply small angle will delete; addition, separate chord deflection method for data reduction, when the adjacent bright spot are in close proximity, even if the string of high small, the angle may be larger, which is not large because the angle be retained. Streamlining process in the cloud of data points of the scan line chord height deviation angle deviation of both aspects limit, can effectively overcome the deficiencies of the above two methods, quickly and efficiently streamline data point cloud.

According to requirements streamline the extent, pre-given the door angle deviation of chords high deviation threshold, the points raised in the two deviation threshold scan line, integrated angle deviation chord height deviation of the two indicators to achieve the objective of streamlining for the line scan point cloud angle - chord streamline the working process of the law shown in Figure 2.

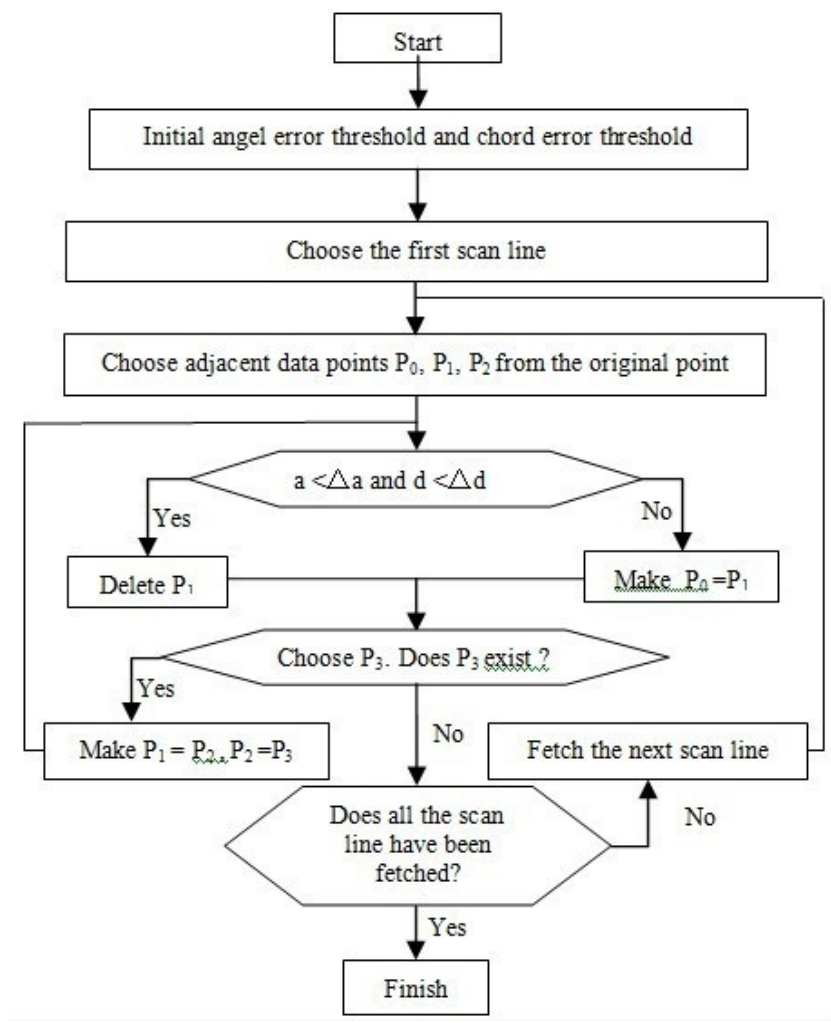

Figure2. Process flow of angle-chord comprehensive simplification method

\section{DATA POINT CLOUD TO STREAMLINE EXAMPLES AND ANALYSIS}

In this paper, the three VIVID910 laser scanner to get the car handle scan line data point cloud, VIVID910 is a noncontact precision color 3D laser scanner, laser beam under the action of light scanning operation, using a slit-like laser on the measurement object is scanned, the reflected light by the CCD 
camera and delicate, and calculating the distance according to the triangulation principle, be obtained by processing the threedimensional data of the measuring object. The car handle Dimensions approximately $95 \mathrm{~mm}$, the surface of the handle by most of the free-form surface, part of the over-surfaces and a small part of the plane, and in order to facilitate the subsequent model reconstruction process, requiring deleted too close to the place in the small curvature of the point cloud data point, but in the point cloud large curvature is retained more frequent data points.

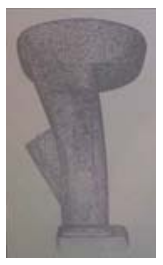

(a)

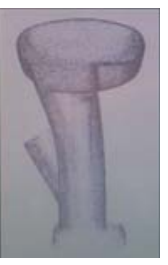

(b)

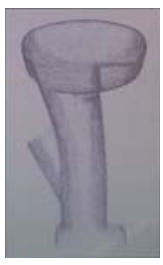

(c)

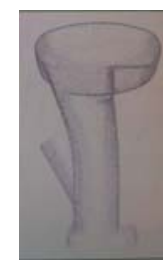

(d)
Figure3. Car handle data point cloud of simplification results

Figure 3 (a) by the point cloud its synthetic surface handle data point cloud, the data point cloud is very dense, about 114,000 data points. Figure 3 (b) to call angle deviation streamlined point cloud, streamlining angle error value of 0.12 (degrees) and streamlined data point cloud about 62,000 data points. Figure 3 (c) is calling the chord deviation method streamlined point cloud, streamlining the chord deviation value of $0.12(\mathrm{~mm})$, and the streamlined data from cloud about 51,000 data points. Figure 3 (d) is a direct call angle - chord comprehensive streamlining streamlined point cloud the streamline angle error value is 0.12 (degrees) the chord deviation value of $0.12(\mathrm{~mm})$, streamlined data from point cloud about 33,000 data points, the point cloud to streamline algorithm to ensure that meet the accuracy requirements, a more streamlined efficiency.

\section{CONCLUSION}

The laser measurement speed, high accuracy, good efficiency, real-time, but the laser measurement point cloud data to obtain a huge amount to the follow-up computing and storage are inconvenient, research data point cloud streamline method has a very important significance. This article describes the common point cloud data point cloud classification and streamlined approach, starting from the analysis of the characteristics of the three-dimensional laser scan data point cloud, based on the angle of curvature change -
--- chord streamline method. The practical application using angle - chord streamline method to the three-dimensional laser scanner scan line data point cloud directly effective and streamlined, and is able to retain more data points, the big change in curvature curve transform small at remove more data points, to better retain the object surface features as reflected in the point cloud of the original data in the case of ensuring streamlined rate.

\section{REFERENCES}

[1]. WANG Qin-feng, HU Zhi-chao, ZHANG Huo-tu, et al. Research and application on the reverse engineering design of automobile panel[J]. Machinery Design \& Manufacture, 2011(4):83-85.

[2]. DONG Ning, PING Xueliang, TAO Yu, et al. Research on the Application of Reverse Engineering Technology in Production of Turbine Blades[J]. MACHINE TOOL \& HYDRAULICS, 2011, 39(15):92-94.

[3]. WANG Ge-fang, CHEN Guo-shun, WANG Xue-ming, et al. Design of Equipment Parts Based on Object Reverse Engineering Technologies[J]. Ordnance Industry Automation, 2009, 28(4):12-14.

[4]. WANG Liangde. Application and Prospect of Reverse Engineering in Designing and Manufacturing Comples Surface Parts[J]. Design an Research, 2009(11):58-60,61.

[5]. MA Hanwei. Disdussion on the Reverse Engineering Technology and Its Application in Industry[J]. Modern manufacture technolofy \& ordnance, 2009(5):24-25.

[6]. XU Ying-xun, DING Hao, ZHU Shi-gen. Key technologies of arbitrary curved surface reverse engineering[J]. Mechanic Transmission, 2006, 30(3):32-35.

[7]. SANSONI G, CORINI S, LAZZARI S. Three-dimensional imaging based on Gray-code light projection: characterization of the measuring algorithm and development of a measuring system of industrial applications[J]. Applied Optics, 2006, 36(19):4463-4472.

[8]. KANG Jian-li, CHEN Gang. Application of the Reverse Engineering in the Localization of the Imported Textile Machinery[J]. Textile Accessories, 2006, 33(1):23-25.

[9]. CHEN Hong-yuan, LIU Dong. Key techniques and latest development in reverse engineering of objects[J]. JOURNAL OF MACHINE DESIGN, 2006, 23(8):1-5.

[10]. ZHAO Yan-Ping, GAO Ming, LUO Da-bing. Data management based on PolyWorks in reverse engineering[J]. Computer Application Technology, 2005, 32(10):38-40.

[11]. XU Zhi-long, FENG Pei-Feng, LI Yan-Ping, et al. Data Collection and Processsing for Complex Curving Curving Surface in Revesre Engineering[J]. Development \& Innovation of machinery \& electrical products, 2005, 18(6):95-97.

[12]. LUO Da-Bing , GAO Ming , WANG Pei-jun. Digitized measurement and point clouds data processing of reverse engineering[J]. Machinery Design \& Manufacture, 2005(9):56-58. 